\title{
Angiogenic Potential of Cryopreserved Amniotic Membrane Is Enhanced Through Retention of All Tissue Components in Their Native State
}

\author{
Yi Duan-Arnold, ${ }^{*}$ Thomas E. Uveges, Alexandra Gyurdieva, \\ Amy Johnson, and Alla Danilkovitch \\ Osiris Therapeutics, Inc., Columbia, Maryland.
}

Objective: Chronic wounds have inadequate microvasculature (or blood vessels), resulting in poor healing. Both fresh human amniotic membrane (hAM) containing viable cells and devitalized hAM have been shown to stimulate angiogenesis in chronic wounds. However, the importance of retaining viable endogenous cells on the angiogenic activity of hAM remains unknown. To understand their role, we compared the angiogenic potential of intact cryopreserved hAM containing viable cells (int-hAM) with devitalized cryopreserved hAM (dev-hAM).

Approach: The effects of conditioned medium (CM) derived from int-hAM and dev-hAM on endothelial cell migration and tube formation were compared. InthAM and dev-hAM CM and tissues were tested for key angiogenic factors, such as vascular endothelial growth factor (VEGF), basic fibroblast growth factor (bFGF), and platelet-derived growth factor-BB (PDGF-BB) after 7 days in culture. The role of VEGF in int-hAM-mediated tube formation was analyzed through inhibition of its activity by anti-VEGF antibody.

Results: CM from int-hAM showed greater endothelial cell recruitment and tube formation compared with dev-hAM. Significantly higher levels of VEGF were detected in int-hAM CM after 1 week compared with dev-hAM CM. InthAM tissue also had significantly greater expression of VEGF and bFGF relative to dev-hAM. A similar trend was observed for PDGF-BB. Neutralization of VEGF in int-hAM CM significantly inhibited tube formation compared with int-hAM CM alone.

Innovation and Conclusion: Preservation of all native hAM components, including viable endogenous cells, enhances the angiogenic effect of cryopreserved hAM. This effect is mediated through higher levels of angiogenic factors, especially VEGF, produced by int-hAM.

\section{INTRODUCTION}

THE NORMAL WOUND-healing process is characterized by three phases: an inflammatory phase, a proliferative phase, and a remodeling phase. ${ }^{1}$ Angiogenesis, or new blood vessel formation, is a key step during the proliferative phase. It re-establishes microvasculature in injured tissues to supply nutrients and oxygen to cells. $^{2}$ Impaired angiogenesis, along with an increased demand for oxygen at the wound site, results in hypoxia, which leads to cell death and poor

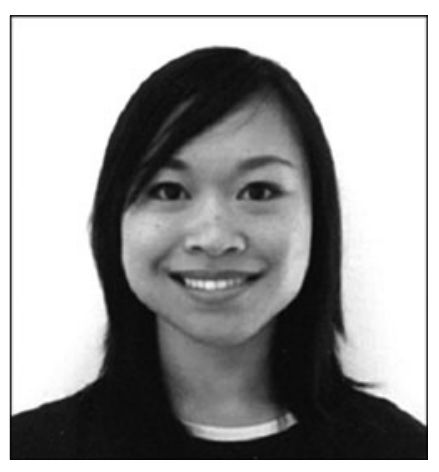

Yi Duan-Arnold, PhD

Submitted for publication January 26, 2015. Accepted in revised form May 9, 2015.

${ }^{*}$ Correspondence: Osiris Therapeutics, Inc. 7015 Albert Einstein Drive, Columbia, MD 21046 (e-mail: yarnold@osiris.com). production in any medium, provided the original author(s) and the source are credited. 
healing. ${ }^{3}$ Studies of chronic wounds have shown that wound exudates have increased levels of antiangiogenic proteins correlating with reduced tubular formation (an in vitro assay of angiogenesis) as compared with acute wounds. ${ }^{4,5}$ Therefore, supplying growth factors to stimulate angiogenesis is a key feature of wound care therapies.

Human amniotic membrane (hAM) was first used to treat wounds in $1910 .{ }^{6}$ One explanation for the success of hAM in healing wounds is its angiogenic potential. ${ }^{7,8}$ Faulk et al. observed new blood vessel formation by histological and immunohistological evaluation of chronic wound biopsies after hAM application. ${ }^{7}$ Despite angiogenic and other beneficial properties of hAM for wound treatment, it was not widely used due to the limited availability of fresh hAM and the risk of disease transmission associated with a short shelf life. ${ }^{9}$ Therefore, a variety of preservation methods have been introduced to overcome these disadvantages of fresh hAM. However, the majority of these methods result in a devitalized tissue.

Although it has been suggested that retention of viable endogenous cells is not important for hAM functionality, we found that maintaining viable endogenous cells significantly enhanced the anti-inflammatory, anti-oxidant, and fibroblast and keratinocyte chemoattractive activities of cryopreserved hAM. ${ }^{10,11}$ In this study, we evaluated the importance of preserving all native components, including viable endogenous cells, on the angiogenic potential of hAM.

\section{CLINICAL PROBLEM ADDRESSED}

Most tissue preservation methods destroy the viable cells within hAM and cause varying degrees of damage to the structural matrix and growth factors. Our data demonstrate that preserving all native tissue components, including viable cells, results in significantly greater angiogenic potential as compared with devitalized human amniotic membrane (dev-hAM).

\section{MATERIALS AND METHODS}

\section{Tissue procurement and ethics statement}

Human term placentas were provided by The National Disease Research Interchange (Philadelphia, PA) and Cord Blood America, Inc. (Las Vegas, NV) from eligible donors after obtaining written informed consent.

\section{Placental tissue processing}

Placental tissues were aseptically processed in a biological safety cabinet within $36 \mathrm{~h}$ after collec- tion. The hAM was separated from the umbilical cord and from the chorion by blunt dissection. Residual blood was removed, and the hAM was incubated in Dulbecco's modified Eagle's medium (DMEM; GE Healthcare Life Sciences, Piscataway, NJ) containing an antibiotic cocktail of gentamicin (Fresenius Kabi USA, Lake Zurich, IL), vancomycin (Hospira, San Jose, CA), and Amphotericin B (Sigma-Aldrich, St. Louis, MO) for $18-48 \mathrm{~h}$ at $37^{\circ} \mathrm{C}$ and $5 \% \mathrm{CO}_{2}$ in a humidified atmosphere. Subsequently, residual antibiotics were removed by washing with Dulbecco's phosphate-buffered saline (DPBS; Life Technologies, Carlsbad, CA), and the hAM was cut into $25 \mathrm{~cm}^{2}$ pieces.

Cryopreservation of hAM was performed by freezing hAM in a dimethyl sulfoxide (Mylan, Inc., Canonsburg, PA) containing cryoprotectant solution at a controlled cooling rate, according to a proprietary process developed by Osiris Therapeutics, Inc. (Columbia, MD). Tissues that were prepared solely by cryopreservation will be referred to as intact human amniotic membrane (int-hAM) in the study. Tissues prepared with cryopreservation and an additional freezing step that resulted in devitalization will be referred to as dev-hAM. Int$\mathrm{hAM}$ and dev-hAM were stored at $-80^{\circ} \mathrm{C}$ for up to 3 months before their use in the experiments. Both int-hAM and dev-hAM were thawed in a room temperature water bath and rinsed with room temperature saline or DPBS before use. Placentas from multiple donors were used in this study.

Samples of fresh placental tissue were prepared as described earlier except that these samples were used immediately for experiments after antibiotic treatment and were not cryopreserved.

\section{Histological evaluation}

Fresh hAM, int-hAM, and dev-hAM samples were fixed in $10 \%$ formalin for $24 \mathrm{~h}$ and then transferred to ethanol. Samples were embedded in paraffin, sectioned into $5 \mu \mathrm{m}$-thick cross-sections, and stained with hematoxylin and eosin (H\&E) according to standard protocols at Histoserv, Inc. (Germantown, MD).

\section{Immunohistological staining}

After being de-paraffinized, rinsed, and blocked with $4 \%$ bovine serum, fresh hAM, int-hAM, and dev-hAM were incubated with primary antibodies against collagen IV (1:500; Abcam, Cambridge, MA) at room temperature overnight, followed by secondary antibodies (1:500) and streptavidinhorseradish peroxidase (1:400; Vector Laboratories, Burlingame, CA), respectively. Stained collagen IV 
was visualized using $3,3^{\prime}$-diaminobenzidine brown stain. Sections were counterstained with hematoxylin (blue nuclei stain). Tissue sections incubated in the absence of primary antibodies served as a negative control.

\section{Assessment of placental tissue cell viability}

A quantitative assessment of the cell viability of tissue samples was performed using the trypan blue exclusion method. Fresh hAM, int-hAM, and dev-hAM were digested in $0.75 \mathrm{mg} / \mathrm{mL}$ collagenase type II solution (Worthington Biochemical Corporation, Lakewood, NJ) in DMEM and incubated with mild agitation at $37^{\circ} \mathrm{C}$ for $30 \mathrm{~min}$. Subsequently, collagenase was removed by centrifugation, and $0.05 \%$ trypsin- ethylenediaminetetraacetic acid (EDTA) solution (Life Technologies) was added to the remaining pellets for further digestion. After 5$10 \mathrm{~min}$, cells released from the tissue were washed with DMEM and pelleted by centrifugation. Cells were counted using a hemocytometer under an inverted microscope (Eclipse TE300; Nikon, Tokyo, Japan), and viable cells were identified by exclusion of the dye trypan blue (Sigma-Aldrich).

For a qualitative assessment of cell viability, the LIVE/DEAD ${ }^{\circledR}$ viability/cytotoxicity kit (Life Technologies) was used according to the manufacturer's instructions. Briefly, tissue samples were incubated in a 1:1,000 dilution of both components of the kit for $30 \mathrm{~min}$. Samples were then visualized under a fluorescent microscope (Eclipse TE300; Nikon). Viable, intact cells within the tissue pieces were identified by green fluorescent calcein AM, whereas dead or damaged cells were labeled with red fluorescent ethidium homodimer-1. Photographs were taken for both green and red channels, which were then merged using ImageJ, a Java ${ }^{\mathrm{TM}}$ based image processing program provided by the National Institute of Health (Bethesda, MD).

To evaluate cell viability after culture for an extended period, int-hAM samples were incubated in DMEM supplemented with $10 \%$ fetal bovine serum (FBS; Life Technologies), 2\% glutamax (Life Technologies), and $2 \%$ antibiotic-antimycotic (Life Technologies) for 7 days. The viability of int-hAM samples was then assessed qualitatively using the LIVE/ $\mathrm{DEAD}$ viability/cytotoxicity kit as described earlier.

\section{Culture of human umbilical vein endothelial cells}

Human umbilical vein endothelial cell (HUVEC; Lonza, Walkersville, MD) were cultured in endothelial growth medium (EGM-2; Lonza) at $37^{\circ} \mathrm{C}$ and $5 \% \mathrm{CO}_{2}$ in a humidified atmosphere until they reached $\sim 80 \%$ confluence. HUVEC at passage 4 and 5 were used in experiments. HUVEC were assayed during their growth phase.

\section{Generation of conditioned medium for functional assays}

Conditioned medium (CM) was generated by incubating int-hAM and dev-hAM in $1 \mathrm{~mL}$ of endothelial basal medium-2 (EBM-2; Lonza) with $2 \%$ FBS (Lonza) for 3 days at $37^{\circ} \mathrm{C}$ and $5 \% \mathrm{CO}_{2}$ in a humidified atmosphere. The CM was collected, centrifuged at $300 \mathrm{~g}$ for $5 \mathrm{~min}$ to remove debris, transferred into new tubes, and stored at $-80^{\circ} \mathrm{C}$ until testing.

\section{HUVEC migration assay}

The ability of hAM to stimulate the migration of HUVEC was determined using the FluroBlok ${ }^{\mathrm{TM}}$ Transwell ${ }^{\circledR}$ system with $8 \mu \mathrm{m}$ pores (BD Biosciences, Franklin Lakes, NJ). Cells were cultured as described earlier, and 50,000 HUVEC in EBM-2 were seeded on top of the porous transwell insert in duplicates and allowed to adhere overnight. CM derived from int-hAM and dev-hAM was added to the lower chambers of the transwells. After overnight incubation, cells that had migrated through the filter were fixed with $4 \%$ paraformaldehyde (USB, Cleveland, $\mathrm{OH}$ ) and stained with CM-DiI (Life Technologies). The samples were analyzed by fluorescent microscopy (Nikon Eclipse TE300; Nikon) at 553/570 nm. The FluoroBlok inserts block transmission of light between 400 and $700 \mathrm{~nm}$, thus enabling quantification of only cells that have migrated to the bottom of the insert. Three images of representative fields were taken of replicate wells at $10 \times$ magnification. For each condition, the number of migrated cells was quantified by three blinded independent operators.

\section{Tube formation assay}

The ability of int-hAM and dev-hAM to stimulate HUVEC to form closed, vessel-like structures was evaluated using a modified protocol described by Arnaoutova and Kleinman. ${ }^{12}$ HUVEC were seeded at a concentration of $1.0 \times 10^{4}$ cells/well on Matrigel (Corning, Corning, NY)-coated culture wells with CM derived from dev-hAM or int-hAM in duplicates as described earlier. HUVEC seeded on Matrigel with EBM-2 and with EGM-2, containing angiogenic growth factors, served as negative and positive controls, respectively. HUVEC cultures were incubated for $6 \mathrm{~h}$ at $37^{\circ} \mathrm{C}$ and $5 \% \mathrm{CO}_{2}$ in a humidified atmosphere and were then stained with calcein AM (Life Technologies). The samples were analyzed by fluorescent microscopy (Eclipse TE300; Nikon), and three images of representative fields were taken of replicate wells at $4 \times$ magnification. For each condition, the number of closed structures was quantified by three blinded independent operators. 
We examined the significance of vascular endothelial growth factor (VEGF) in mediating tube formation by neutralizing its activity. CM derived from int-hAM was incubated alone or with $50 \mu \mathrm{g} /$ $\mathrm{mL}$ anti-VEGF ${ }_{165}$ antibody (R\&D Systems, Minneapolis, MN) for $1 \mathrm{~h}$ at $37^{\circ} \mathrm{C}$ and $5 \% \mathrm{CO}_{2}$ in a humidified atmosphere. Goat IgG isotype control (R\&D Systems) in the same conditions at $50 \mu \mathrm{g} / \mathrm{mL}$ was used as a control. These samples were tested in the in vitro tube formation assay described earlier.

\section{Analysis of angiogenic growth factors in hAM}

The presence of three key angiogenic growth factors in hAM was evaluated: VEGF, basic fibroblast growth factor (bFGF), and platelet-derived growth factor-BB (PDGF-BB). Int-hAM and devhAM were incubated in $1 \mathrm{~mL}$ of DMEM (GE Healthcare Life Sciences) with 10\% FBS (Life Technologies), 2\% glutamax (Life Technologies), and $2 \%$ antibiotic-antimycotic (Life Technologies) for 7 days at $37^{\circ} \mathrm{C}$ and $5 \% \mathrm{CO}_{2}$ in a humidified atmosphere. CM was centrifuged at $300 \mathrm{~g}$ for $5 \mathrm{~min}$, and supernatants were collected and stored at $-80^{\circ} \mathrm{C}$ before analysis. For VEGF and PDGF-BB testing, $250 \mu \mathrm{L} \mathrm{CM}$ was incubated with $250 \mu \mathrm{L} 8 \mathrm{M}$ guanidine $\mathrm{HCl}(\mathrm{GuHCl}$; Thermo Fisher Scientific, Waltham, MA) containing $10 \mathrm{mM}$ calcium chloride $\left(\mathrm{CaCl}_{2} ;\right.$ Sigma-Aldrich), $50 \mathrm{mM}$ Tris (SigmaAldrich), and protease inhibitor (Roche, Basel, Switzerland) at $4^{\circ} \mathrm{C}$ overnight with rotation. Samples were centrifuged at $300 \mathrm{~g}$ for $10 \mathrm{~min}$ at $4^{\circ} \mathrm{C}$ and then desalted using Zeba Desalting Columns (Thermo Fisher Scientific) as per the manufacturer's instructions. Desalted samples were collected and stored at $-80^{\circ} \mathrm{C}$ until further testing. The Guanidine $\mathrm{HCl}$ treatment of samples before measurement of VEGF and PDGF-BB was performed to eliminate factors that interfere with VEGF and PDGF-BB detection by enzyme-linked immunosorbent assay (ELISA). The culture medium (DMEM with $10 \%$ FBS) was included in ELISAs as a baseline control for CM.

For the evaluation of angiogenic growth factors at day 0 and after 7 days in culture, tissue lysates (TL) were prepared as described next.

For extraction of bFGF, tissue samples of inthAM and dev-hAM were lysed in Tissue Protein Extraction Reagent (Thermo Scientific, Waltham, MA) containing protease inhibitor (Roche). Briefly, tissue was removed from $-80^{\circ} \mathrm{C}$, and $0.5 \mathrm{~mL}$ of lysis buffer with 1 pre-chilled $5 \mathrm{~mm}$ stainless steel bead (Qiagen, Venlo, Limburg) was added to the cold tube. The tissue was then lysed for $6 \mathrm{~min}$ at $50 \mathrm{~Hz}$ in the pre-chilled chamber of the TissueLyser LT (Qiagen) and centrifuged at $300 \mathrm{~g}$ for $15 \mathrm{~min}$ at $4^{\circ} \mathrm{C}$ to remove debris. Supernatants were collected and stored at $-80^{\circ} \mathrm{C}$ until further testing.

For extraction of VEGF and PDGF-BB, tissue samples of int-hAM and dev-hAM were lysed in $0.5 \mathrm{~mL}$ of $8 \mathrm{M} \mathrm{GuHCl}$ (Thermo Scientific) containing $10 \mathrm{mM} \mathrm{CaCl}_{2}$ (Sigma-Aldrich), $50 \mathrm{mM}$ Tris (Sigma-Aldrich), and protease inhibitor (Roche). Briefly, tissue was homogenized in GuHCl lysis buffer using TissueLyser LT as described earlier (Qiagen). Subsequently, TL were incubated at $4^{\circ} \mathrm{C}$ overnight with rotation, centrifuged at $300 \mathrm{~g}$ for $10 \mathrm{~min}$ at $4^{\circ} \mathrm{C}$, and desalted using Zeba Desalting Columns (Thermo Scientific) as per the manufacturer's instructions. Desalted TL were collected and stored at $-80^{\circ} \mathrm{C}$ until further testing.

TL and CM were tested for VEGF by Quantikine ELISA (R\&D Systems) and for PDGF-BB and bFGF by DuoSet ELISA (R\&D Systems). Total levels of VEGF, bFGF, and PDGF-BB for int-hAM and for dev-hAM were calculated as a sum of levels of these growth factors detected in CM and TL.

\section{Statistical analysis}

Data are presented as mean \pm standard deviation for one representative experiment with two replicates. Student's $t$-test was used for statistical analysis, and $p<0.05$ was considered significant.

\section{RESULTS \\ Comparison of int-hAM and dev-hAM composition}

The structural and cellular integrity of int-hAM and dev-hAM were compared to confirm that the only difference between int-hAM and dev-hAM was the viability of endogenous cells. $\mathrm{H} \& \mathrm{E}$ and collagen IV staining demonstrated that the tissue matrix in both int-hAM and dev-hAM was preserved in a similar manner to fresh hAM (Fig. 1A-F). The viability of int-hAM and dev-hAM post-thaw was examined using the LIVE/DEAD viability/cytotoxicity assay. The viability of endogenous cells in int-hAM is similar to fresh hAM (Fig. 2). Trypan blue exclusion assay was performed to confirm that there were on average $84 \% \pm 3 \%$ and $82 \% \pm 6 \%$ viable cells in fresh hAM and int-hAM, respectively. As expected, freezing and cryopreservation resulted in only dead cells in dev-hAM post-thaw (Fig. 2). In addition, the majority of cells in inthAM remained viable after 7 days in culture (Fig. 2G, H).

\section{Recruitment of endothelial cells by int-hAM and dev-hAM}

Chronic wounds have compromised microvasculature, resulting in hypoxia and consequent cell 

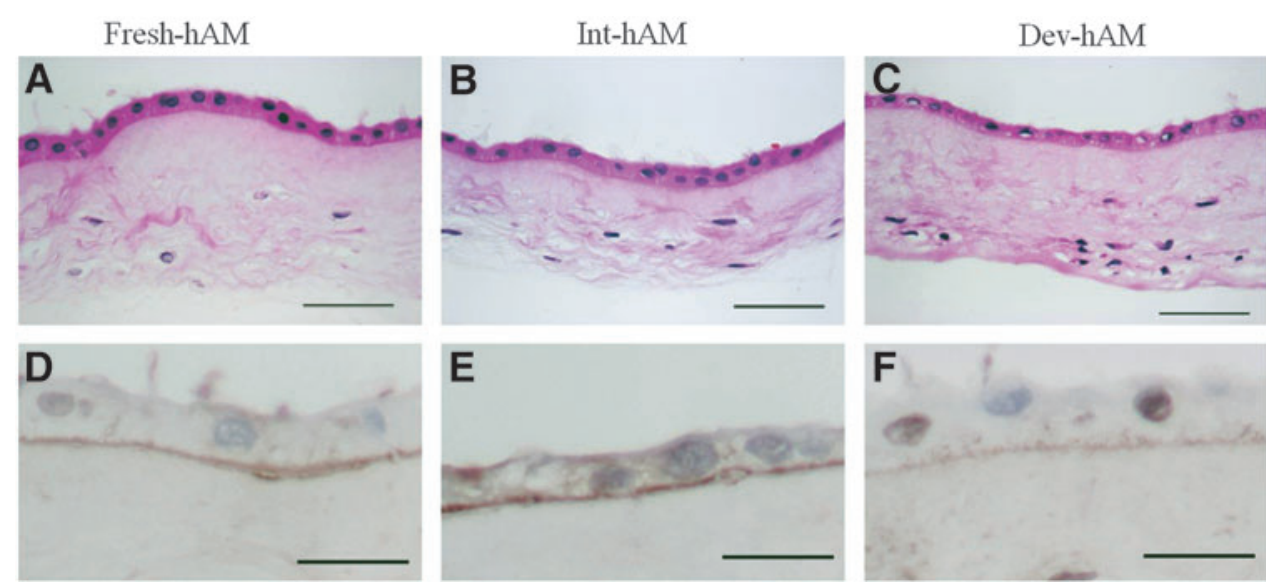

Figure 1. Hematoxilin \& eosin (H\&E) and collagen IV (Col IV) staining of human amniotic membranes (hAM). H\&E staining of (A) fresh hAM, (B) viable intact cryopreserved hAM (int-hAM), and (C) devitalized cryopreserved hAM (dev-hAM). Brown colored stained Col IV in the basement membrane of (D) fresh-hAM, (E) int-hAM, and (F) dev-hAM. Scale bars: $50 \mu \mathrm{m}$ (H\&E) and $20 \mu \mathrm{m}$ (Col IV).

death that prevents wound healing. ${ }^{3,13}$ One of the first steps of new blood vessel formation is recruitment of endothelial cells. ${ }^{14}$ The ability of hAM to recruit endothelial cells was tested using an in vitro cell migration assay. In this assay, HUVEC seeded in the upper chamber of a transwell insert containing a porous membrane migrated to the underside of the filter in response to chemoattractants, such as growth factors (e.g., VEGF; Fig. 3 , positive control). In the absence of chemoattractants, HUVEC remained on the top side of the filter (i.e., non-migratory; Fig. 3, negative control). CM derived from int-hAM induced the migration of $36 \pm 2$ cells per field, while dev-hAM induced the migration of $21 \pm 1$ cells per field (Fig. 3 ). This in- dicates that both $\mathrm{CM}$ contain endothelial cell chemoattractive factors; however, CM derived from int-hAM showed a significantly higher magnitude of effect relative to dev-hAM (68\% higher).

\section{Induction of HUVEC tube formation by int-hAM and dev-hAM}

After recruitment of endothelial cells to the wound site, the proper combination of signaling molecules (e.g., growth factors) is required to trigger formation of new blood vessels by endothelial cells. ${ }^{14}$ The ability of CM derived from int-hAM and dev-hAM to stimulate angiogenesis was evaluated by an in vitro tube formation assay. CM derived from int-hAM induced the formation of $34 \pm 7$
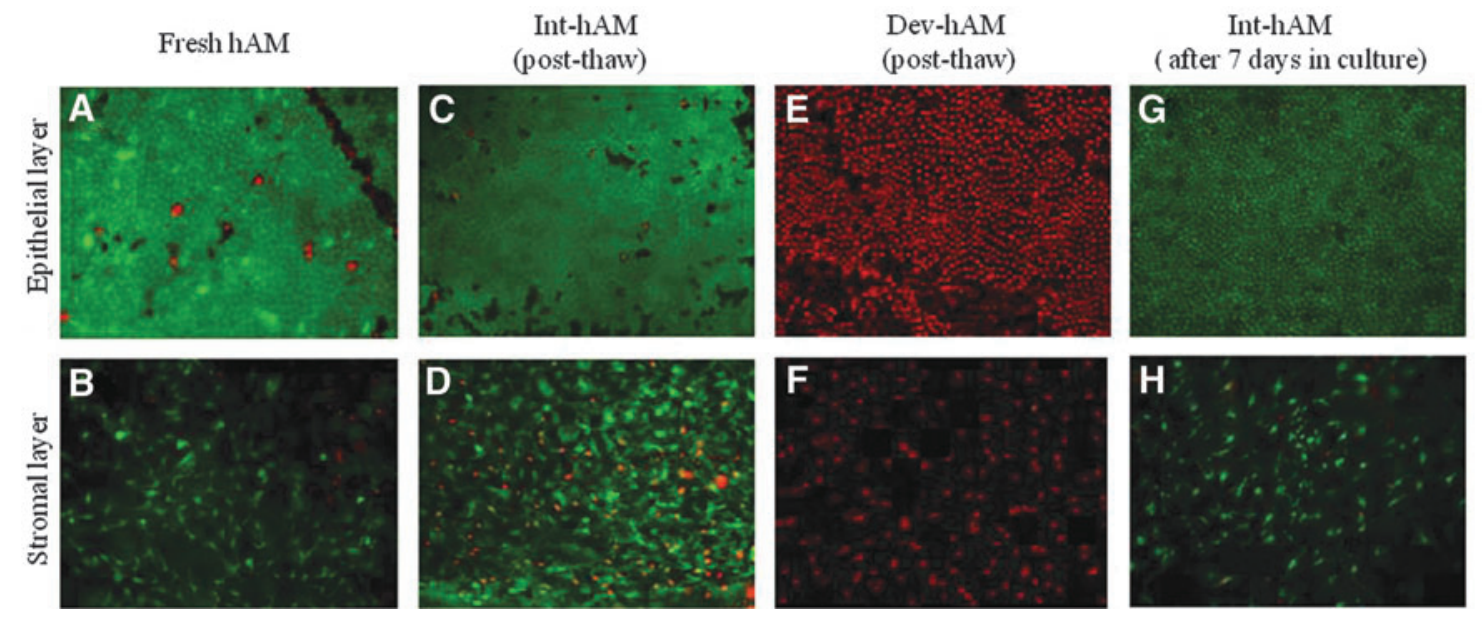

Figure 2. Live and dead endogenous cell staining of hAM. Live and dead cells in epithelial and stromal layers of fresh hAM (A, B), int-hAM post-thaw (C, D), and dev-hAM post-thaw (E, F) were visualized microscopically using the LIVE/DEAD viability/cytotoxicity kit. Live cells stained green with calcein AM. Dead cells stained red with ethidium homodimer-1. Live and dead cells in int-hAM after 7 days in culture are shown in (G) and (H). All images were taken at $10 \times$ magnification. 

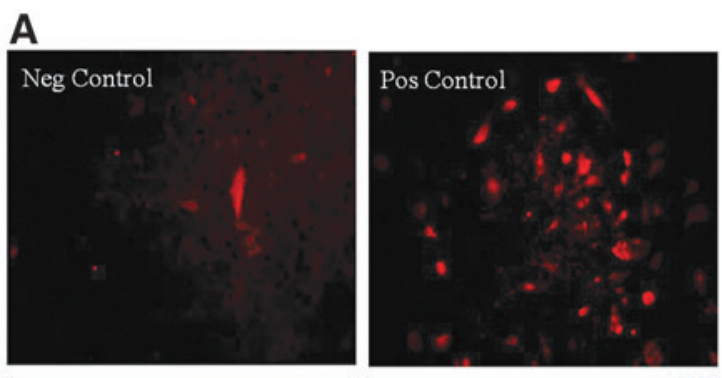

B
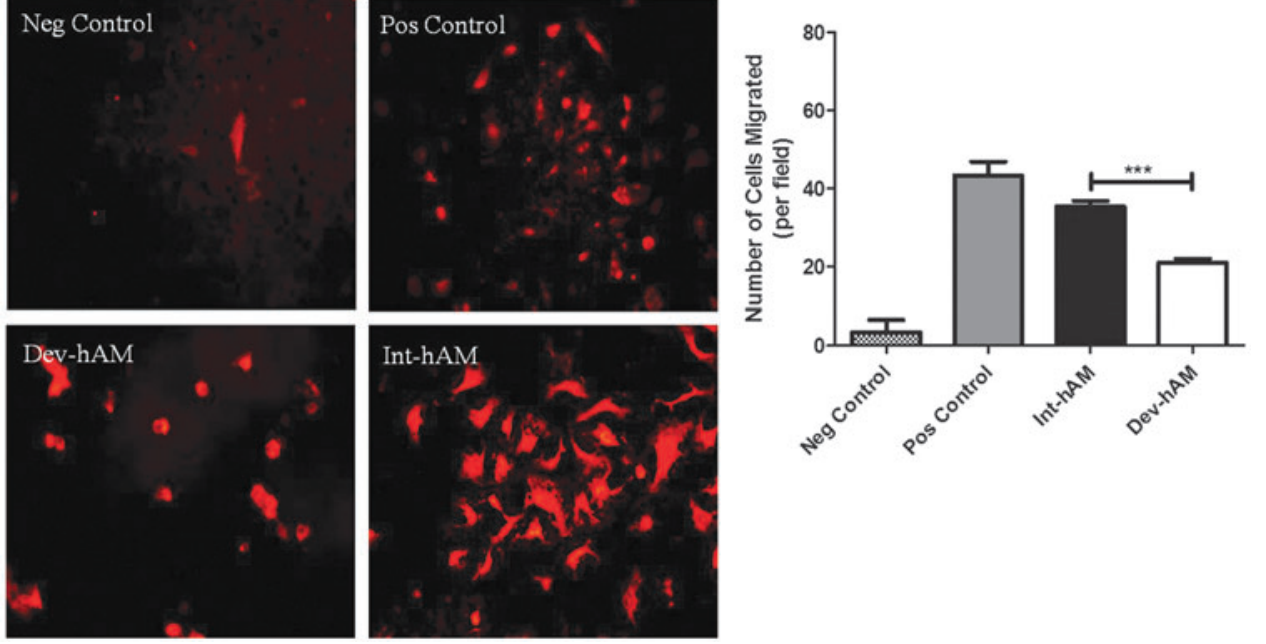

Figure 3. Effect of hAM on human umbilical vein endothelial cells (HUVEC) migration. The migration of HUVEC induced by conditioned medium (CM) derived from int-hAM and dev-hAM was tested. HUVEC were incubated with CM overnight, and migrated HUVEC were stained with CM-Dil and visualized microscopically. The number of migrated HUVEC was counted, and results are presented as the number of cells migrated per microscopic field. Basal medium was used as a negative (Neg) control, and HUVEC growth medium was used as a positive (Pos) control. Representative images of migrated HUVEC at $10 \times$ magnification are shown in (A). Quantitative results are shown in (B). Data are presented as mean \pm standard deviation (SD) for one representative experiment out of three, with two biological replicates counted by three blinded operators. Student's $t$-test was used for statistical analysis. ${ }^{* * *} p<0.001$.

closed structures per field (Fig. 4). This number of closed structures is comparable to that of HUVEC cultured in the presence of angiogenic growth factors (positive control), which had an average of $38 \pm 5$ closed structures per field (Fig. 4). HUVEC cultured with CM derived from dev-hAM formed $4 \pm 3$ closed structures, which was similar to HUVEC cultured in the absence of growth factors (negative control; Fig. 4). Int-hAM showed a $742 \%$ increase in the number of tubular structures relative to dev-hAM.

\section{Angiogenic growth factors in int-hAM and dev-hAM}

VEGF, bFGF, and PDGF-BB are three key angiogenic factors. ${ }^{13}$ To evaluate the release of growth factors by int-hAM and dev-hAM, tissues were either lysed right after thawing or placed into
A
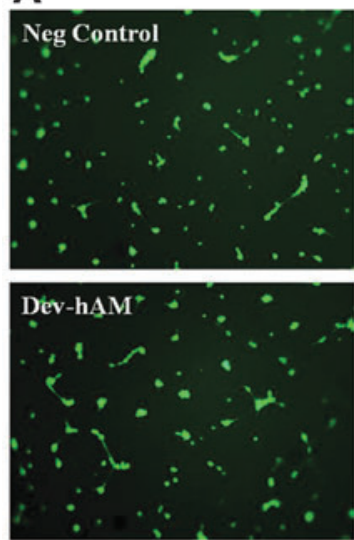
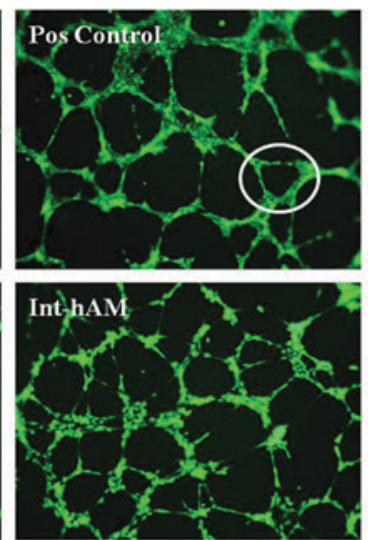

B

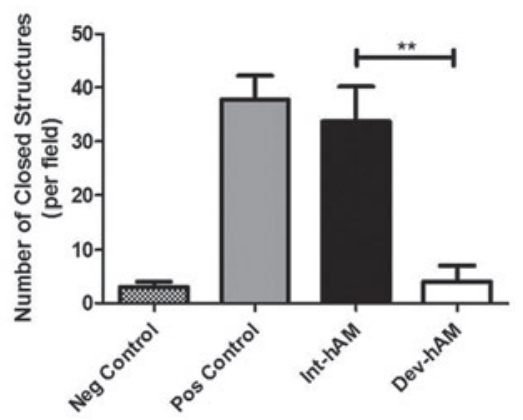

Figure 4. Effect of hAM on HUVEC tube formation. Formation of closed, vessel-like structures by HUVEC was evaluated by culturing cells for $6 \mathrm{~h}$ with CM derived from int-hAM and dev-hAM. HUVEC were then labeled with calcein AM and visualized microscopically. The number of closed, tubular structures (an example of which is labeled with a white circle in Fig. 2A, positive control) formed by HUVEC was counted, and results are presented as the number of closed structures per microscopic field. Basal medium was used as a negative (Neg) control, and HUVEC growth medium was used as a positive (Pos) control. Representative images of HUVEC at $4 \times$ magnification are shown in (A). Results of quantitative analysis are shown in (B). Data are presented as mean \pm SD for one representative experiment out of three, with two biological replicates counted by three blinded operators. Student's $t$-test was used for statistical analysis. ${ }^{* *} p<0.01$. 

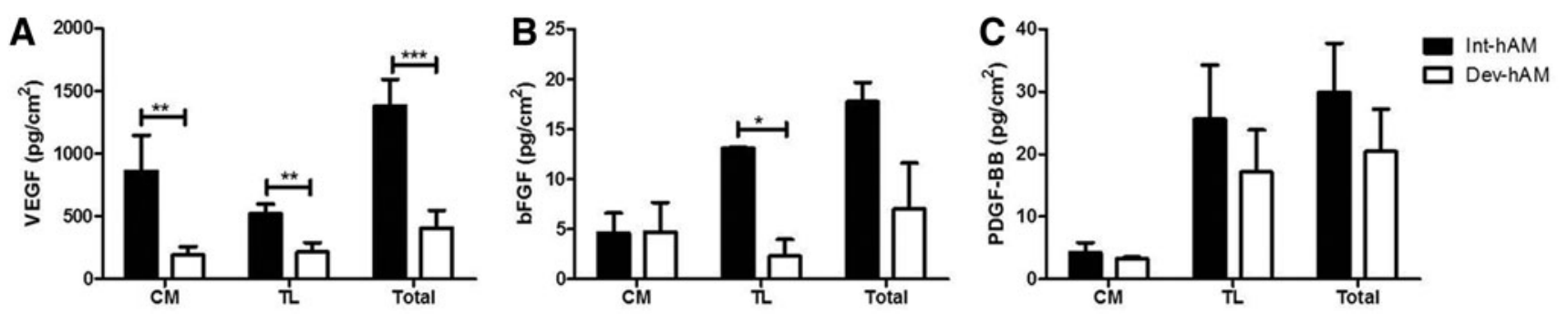

Figure 5. Levels of angiogenic growth factors in hAM after 7 days in culture. int-hAM and dev-hAM were cultured for 7 days. The CM and tissue lysates (TL) were collected and tested for vascular endothelial growth factor (VEGF) (A), basic fibroblast growth factor (bFGF) (B), and platelet-derived growth factor-BB (PDGF-BB) (C) by ELISA. The sum of growth factor content in CM and TL represents total values. Data are presented as mean \pm SD for one representative experiment out of three, with two biological replicates. Student's $t$-test was used for statistical analysis. ${ }^{*} p<0.05,{ }^{* *} p<0.01$, and ${ }^{* * *} p<0.001$.

culture for 7 days, and the growth factor levels in CM and the TL were quantified by ELISA. There was no difference in these angiogenic growth factors between int-hAM and dev-hAM at day 0 postthaw. All values were within the following ranges: VEGF: $110 \pm 40 \mathrm{pg} / \mathrm{cm}^{2}$; bFGF: $18 \pm 15 \mathrm{pg} / \mathrm{cm}^{2}$; and PDGF-BB: $11 \pm 4 \mathrm{pg} / \mathrm{cm}^{2}$. The culture medium containing $10 \%$ FBS, which was used for generation of CM, showed no detectable levels of VEGF, bFGF, or PDGF-BB. Int-hAM had significantly greater levels of VEGF in both CM and TL relative to dev-hAM $\left(861 \pm 288 \mathrm{pg} / \mathrm{cm}^{2}\right.$ vs. $191 \pm 66 \mathrm{pg} / \mathrm{cm}^{2}$ in $\mathrm{CM}$ and $521 \pm 78 \mathrm{pg} / \mathrm{cm}^{2}$ vs. $214 \pm 77 \mathrm{pg} / \mathrm{cm}^{2}$ in TL, Fig. 5A). The level of bFGF in int-hAM TL was significantly greater compared with dev-hAM $\left(13 \pm 0.05 \mathrm{pg} / \mathrm{cm}^{2}\right.$ vs. $2 \pm 2 \mathrm{pg} / \mathrm{cm}^{2}$, Fig. $\left.5 \mathrm{~B}\right)$. The levels of PDGF-BB trended higher in both CM and TL derived from int-hAM as opposed to dev-hAM but did not reach statistical significance $(4 \pm 2 \mathrm{pg} /$ $\mathrm{cm}^{2}$ vs. $3 \pm 0.3 \mathrm{pg} / \mathrm{cm}^{2}$ in $\mathrm{CM}$ and $26 \pm 9 \mathrm{pg} / \mathrm{cm}^{2}$ vs. $17 \pm 7 \mathrm{pg} / \mathrm{cm}^{2}$ in TL, Fig. 5C). The total levels of factors detected in $\mathrm{CM}$ and TL combined were
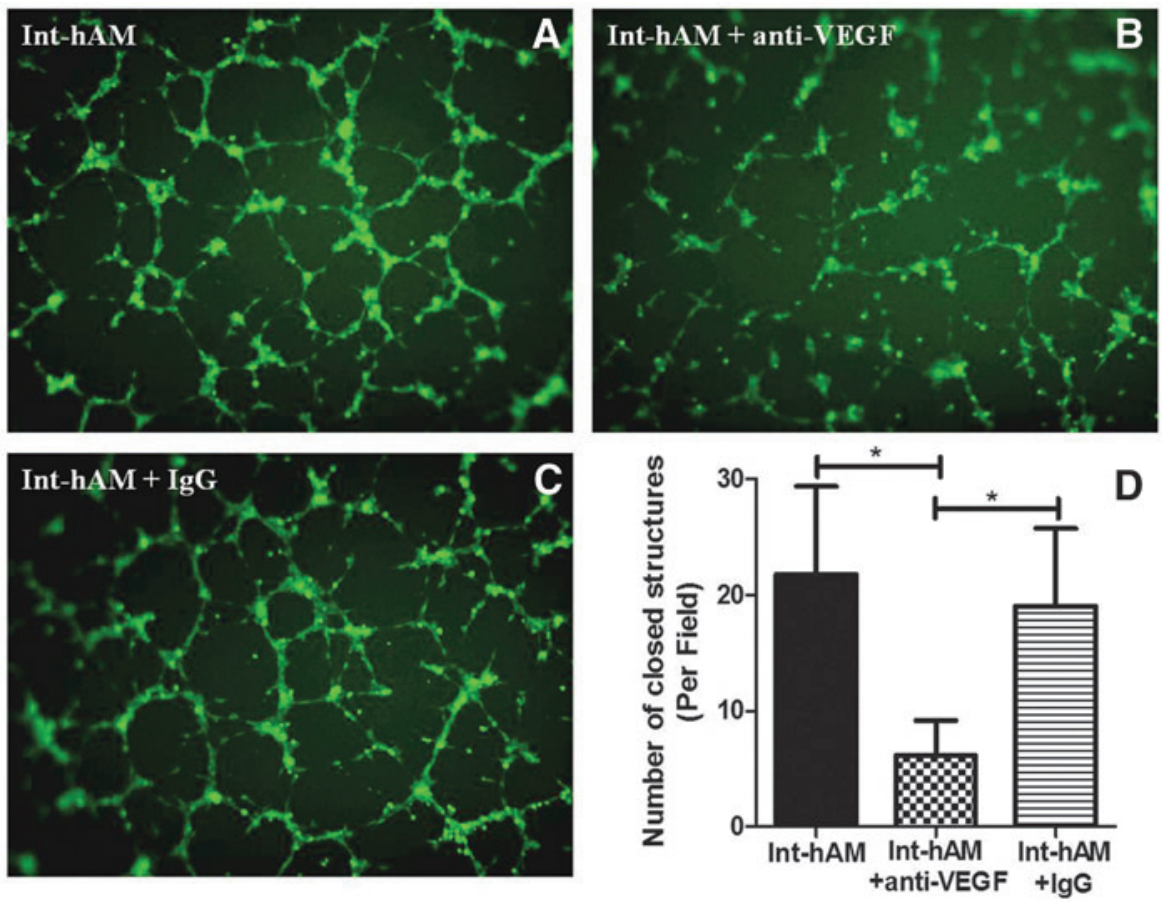

Figure 6. Anti-VEGF neutralizing antibody blocked tube formation induced by CM derived from int-hAM. Int-hAM CM alone (A), or with $50 \mu \mathrm{g} / \mathrm{mL}$ anti-VEGF 165 antibody (B), or with $50 \mu \mathrm{g} / \mathrm{mL}$ goat IgG control (C) were used in vitro tube formation assay. The number of closed, tubular structures was counted, and results are presented as the number of closed structures per microscopic field. Photographs of HUVEC were taken at $4 \times$ magnification. Results of quantitative analysis are shown in (D). Data are presented as mean \pm SD for one representative experiment out of two, with two biological replicates counted by three blinded operators. Student's $t$-test was used for statistical analysis. ${ }^{*} p<0.05$. 
greater for VEGF $(p<0.001), \operatorname{bFGF}(p=0.09)$, and PDGF-BB $(p=0.05)$ as compared with dev-hAM.

\section{Importance of VEGF released by int-hAM for tube formation}

To evaluate the biological significance of increased VEGF levels in int-hAM CM, we added anti-VEGF neutralizing antibody to int-hAM CM and performed an in vitro tube formation assay. Results showed that CM derived from int-hAM induced the formation of $22 \pm 8$ closed structures per field; however, this decreased significantly to $6 \pm 3$ closed structures per field in the presence of anti-VEGF antibody $(p<0.05$; Fig. 6$)$. When IgG isotype control was added to int-hAM CM, $19 \pm 7$ closed structures per field were observed, which was not statistically different from the number of closed structures induced by int-hAM CM alone (Fig. 6).

\section{DISCUSSION}

Angiogenesis, or blood vessel formation, is a dynamic process that is orchestrated by a variety of growth factors and other mediators. ${ }^{13}$ Impaired angiogenesis, a characteristic of chronic wounds, leads to hypoxia, and, consequently, to poor healing. Wound oxygenation is an important prognostic parameter that physicians often measure to predict treatment outcomes. ${ }^{3}$

Fresh and processed devitalized placental membranes have been shown to have angiogenic properties, such as promotion of microvessel formation and recruitment of hematopoietic progenitor cells, which are beneficial for wound treatment. ${ }^{7,15,16}$ Placental membrane processing and preservation is necessary for the development of commercial products for clinical use. Most commercial placental products are devitalized, but the value of maintaining viable cells has not been addressed. In this study, we evaluated the contribution of endogenous viable cells to the angiogenic activity of placental tissues by comparing int-hAM, containing viable cells, and dev-hAM. Specifically, we evaluated the ability of int-hAM and dev-hAM to mediate endothelial cell migration and tube formation, as well as their ability to release angiogenic growth factors in vitro. To devitalize hAM, a freezing step was introduced. Histological and cell viability testing confirmed that the structural matrix in int-hAM and dev-hAM was preserved (Fig. 1), and that only int-hAM retained viable cells (Fig. 2). Retention of viable cells resulted in significantly greater angiogenic activity in int-hAM compared with dev-hAM: a $68 \%$ increase in endo-

\section{KEY FINDINGS}

- The presence of endogenous viable cells enhances the angiogenic activity of cryopreserved hAM.

- Int-hAM has a stronger effect on endothelial cell migration and tube formation relative to dev-hAM, which may be due in part to a significantly higher levels of soluble VEGF released from int-hAM.

thelial cell migration and a $742 \%$ increase in endothelial tube formation in vitro.

Growth factors such as VEGF, bFGF, and PDGF-BB are key angiogenic factors required for endothelial cell migration and new blood vessel formation. ${ }^{12}$ These three angiogenic growth factors work in synergy to promote endothelial cell migration and blood vessel formation. ${ }^{13,17-19}$ Studies have suggested that bFGF is the initial angiogenic stimulus in wound repair, followed by increased expression of VEGF. ${ }^{20}$ When applied topically to diabetic wounds, VEGF led to increased angiogenesis and wound healing. ${ }^{21} \mathrm{PDGF}$ is a multifunctional growth factor with three isoforms: PDGF-AA, $\mathrm{AB}$, and $\mathrm{BB}$. Angiogenic activities of PDGF include new vessel formation (specifically PDGF-BB) and recruitment of periendothelial cells to stabilize the newly formed vessels. ${ }^{14,22}$

Therefore, we evaluated the presence and release of VEGF, bFGF, and PDGF-BB in int-hAM and dev-hAM. Of these three angiogenic growth factors, significantly higher levels of VEGF (350\% increase) were detected in CM derived from inthAM relative to dev-hAM. This suggested that higher magnitude of int-hAM angiogenic activity might be mediated by higher levels of VEGF detected in int-hAM-derived CM. To confirm this hypothesis, we added an anti-VEGF neutralizing antibody to int-hAM CM and performed the tube formation assay. Blocking VEGF activity in inthAM CM significantly decreased the number of closed structures as compared with $\mathrm{CM}$ alone (72\% decrease). This result is consistent with other studies that showed angiogenesis is inhibited by addition of a VEGF neutralizing antibody. ${ }^{23}$ Our data also suggest that dev-hAM may not have sufficient levels of VEGF or the required ratio between VEGF and other growth factors to support tube formation.

The increase in growth factor levels in CM derived from int-hAM may be due to continued growth factor production by endogenous viable cells, which are preserved in int-hAM. Studies have shown that hAM and hAM-derived cells are a source of VEGF, bFGF, and other angiogenic 
growth factors. ${ }^{24,25}$ In addition to $\mathrm{CM}$, we also tested the presence of VEGF, bFGF, and PDGF-BB in TL, because extracellular matrix (ECM) is known to serve as a growth factor reservoir. ${ }^{26}$ The levels of VEGF and bFGF were significantly greater in TL of int-hAM as compared with devhAM. The values of growth factors observed in TL of dev-hAM are similar to previously published results. ${ }^{27}$ When $\mathrm{CM}$ and TL were added together to obtain total values, only VEGF was significantly higher in int-hAM as compared with dev-hAM.

Taken together, our results demonstrate that int-hAM has higher angiogenic activity as compared with dev-hAM: int-hAM induced significantly greater migration and tube formation of endothelial cells, which could be due to the significantly greater level of VEGF in CM as compared with dev-hAM. Previously, we showed that retention of endogenous viable cells increases antioxidant, fibroblast, and keratinocyte chemoattractive activities of cryopreserved hAM. ${ }^{11}$ This study shows the importance of preserving all tissue components native to hAM, including endogenous viable cells, for the angiogenic activity of hAM.

This study has its limitations. HUVEC were used to perform the tube formation and cell migration assays. While there are similarities between different types of endothelial cells, the use of dermal microvascular cells could be more relevant to in vivo responses to hAM when it would be applied to cutaneous wounds. Experiments in which dermal microvascular cells are employed should be the focus of future studies. Second, this study includes only in vitro experiments. However, results of this study give insight into the angiogenic activity of int-hAM and are an appropriate first step to be followed by future in vivo studies. For example, these results would be confirmed by using int-hAM in a diabetic mouse model to mimic an impaired wound-healing environment.

\section{INNOVATION}

Placental membranes, especially hAM, are known to promote angiogenesis. ${ }^{7}$ However, the value of preserving hAM endogenous cells on the angiogenic potential of hAM remains unclear. In this study, we demonstrated that viable endogenous cells enhanced the angiogenic activity of cryopreserved hAM as compared with dev-hAM. This may be due to release of higher levels of VEGF by int-hAM in comparison to dev-hAM.

\section{ACKNOWLEDGMENT AND FUNDING SOURCE}

This research was supported by Osiris Therapeutics, Inc.

\section{AUTHOR DISCLOSURE AND GHOSTWRITING}

All listed authors are employees of Osiris Therapeutics, Inc. This article was not written by any writer other than the authors.

\section{ABOUT THE AUTHORS}

Yi Duan-Arnold, PhD, is currently a senior scientist at Osiris Therapeutics, Inc (OTI). She obtained her $\mathrm{PhD}$ in Biomedical Engineering from City College of New York and followed by a postdoctoral training at Yale Medical School. After that, she worked at the Laboratory for Stem Cells and Tissue Engineering at Columbia University on cardiac tissue engineering. Thomas E. Uveges, $\mathbf{P h D}$, is an associate director at OTI. Alexandra Gyurdieva, MS is a scientist at OTI. Amy Johnson, BS is a senior research associate at OTI. Alla Danilkovitch, $\mathbf{P h D}$, is currently the Chief Scientific Officer at OTI. Dr. Danilkovitch has more than 25 years of a broad biomedical research experience, including stem cell biology, immunology, and cancer research. Dr. Danilkovitch earned a $\mathrm{PhD}$ degree in cell biology.

\section{REFERENCES}

1. Clark R. Overview and general considerations of wound repair. In: Clark RAF, Henson PM, eds. The Molecular and Cellular Biology of Wound Repair. Springer, Science \& Business Media, New York; 1988:3-33.

2. Falanga $V$. Wound healing and its impairment in the diabetic foot. Lancet 2005;366:1736-1743.

3. Sen CK. Wound healing essentials: let there be oxygen. Wound Repair Regen 2009;17:1-18.

4. Ulrich D, Lichtenegger F, Unglaub F, Smeets R, Pallua N. Effect of chronic wound exudates and
MMP-2/-9 inhibitor on angiogenesis in vitro. Plast Reconstruct Surg 2005;116:539-545.

5. Krisp C, Jacobsen F, McKay MJ, Molloy MP, Steinstraesser L, Wolters DA. Proteome analysis reveals antiangiogenic environments in chronic wounds of diabetes mellitus type 2 patients. Proteomics 2013;13:2670-2681.

6. Davis JW. Skin transplantation with a review of 550 cases at the Johns Hopkins Hospital. Johns Hopkins Med J 1910;15:307.
7. Faulk WP, Matthews R, Stevens PJ, Bennett JP, Burgos H, Hsi BL. Human amnion as an adjunct in wound healing. Lancet 1980;1:11561158

8. Egan TJ, O'Driscoll J, Thakar DR. Human amnion in the management of chronic ulceration of the lower limb: a clinico-pathologic study. Angiology 1983;34:197-203.

9. Adds PJ, Hunt CJ, Dart JK. Amniotic membrane grafts, "fresh" or frozen? A clinical and 
in vitro comparison. $\mathrm{Br} \mathrm{J}$ Ophthalmol 2001;85 905-907.

10. Duan-Arnold Y, Gyurdieva A, Johnson A, Uveges T, Jacobstein DA, Danilkovitch-Miagkova A. Retention of endogenous viable cells enhances the anti-inflammatory activity of cryopreserved amnion. Adv Wound Care 2015 (in press).

11. Duan-Arnold Y, Gyurdieva A, Johnson A, Jacobstein DA, Danikovitch A. Soluble factors released by endogeneous viable cells enhance the antioxidant and chemoattractive activities of cryopreserved amnionotic membrane. Adv Wound Care 2015;4(6):329-338.

12. Arnaoutova I, Kleinman HK. In vitro angiogenesis: endothelial cell tube formation on gelled basement membrane extract. Nature protocols 2010;5: 628-635.

13. Cui T, Kirsner R, Li J. Angiogenesis in chronic wounds. Adv Wound Care 2010;1:347-352.

14. Conway EM, Collen D, Carmeliet P. Molecular mechanisms of blood vessel growth. Cardiovasc Res 2001:49:507-521.

15. Koob TJ, Lim JJ, Massee M, et al. Angiogenic properties of dehydrated human amnion/chorion allografts: therapeutic potential for soft tissue repair and regeneration. Vasc Cell 2014;6:10

16. Maan ZN, Rennert RC, Koob TJ, Januszyk M, Li WW, Gurtner GC. Cell recruitment by amnion chorion grafts promotes neovascularization. J Surg Res 2015;193:953-962.

17. Asahara T, Bauters C, Zheng LP, et al. Synergistic effect of vascular endothelial growth factor and basic fibroblast growth factor on angiogenesis in vivo. Circulation 1995;92:||365-||371.
18. Cao R, Brakenhielm E, Pawliuk R, et al. Angiogenic synergism, vascular stability and improvement of hind-limb ischemia by a combination of PDGF-BB and FGF-2. Nat Med 2003:9:604-613.

19. Kano MR, Morishita Y, Iwata C, et al. VEGF-A and FGF-2 synergistically promote neoangiogenesis through enhancement of endogenous PDGF-BPDGFRbeta signaling. J Cell Sci 2005;118:37593768.

20. Nissen NN, Polverini PJ, Koch AE, Volin MV Gamelli RL, DiPietro LA. Vascular endothelial growth factor mediates angiogenic activity during the proliferative phase of wound healing. Am J Pathol 1998;152:1445-1452.

21. Galiano RD, Tepper OM, Pelo CR, et al. Topical vascular endothelial growth factor accelerates diabetic wound healing through increased angiogenesis and by mobilizing and recruiting bone marrow-derived cells. Am J Pathol 2004;164: 1935-1947.

22. Battegay EJ, Rupp J, Iruela-Arispe L, Sage EH, Pech M. PDGF-BB modulates endothelial proliferation and angiogenesis in vitro via PDGF betareceptors. J Cell Biol 1994;125:917-928.

23. Seghezzi G, Patel S, Ren CJ, et al. Fibroblast growth factor-2 (FGF-2) induces vascular endothelial growth factor (VEGF) expression in the endothelial cells of forming capillaries: an autocrine mechanism contributing to angiogenesis. J Cell Biol 1998;141:1659-1673.

24. Yamahara K, Harada K, Ohshima M, et al. Comparison of angiogenic, cytoprotective, and immunosuppressive properties of human amnion- and chorion-derived mesenchymal stem cells. PLoS One 2014;9:e88319.
25. Lopez-Valladares MJ, Teresa Rodriguez-Ares $M$ Tourino R, Gude F, Teresa Silva M, Couceiro J. Donor age and gestational age influence on growth factor levels in human amniotic membrane. Acta Ophthalmol 2010;88:e211-e216.

26. Li J, Zhang YP, Kirsner RS. Angiogenesis in wound repair: angiogenic growth factors and the extracellular matrix. Microsc Res Tech 2003;60:107-114.

27. Russo A, Bonci P, Bonci P. The effects of different preservation processes on the total protein and growth factor content in a new biological product developed from human amniotic membrane. Cell Tissue Bank 2012;13:353-361.

$$
\begin{aligned}
& \text { Abbreviations and Acronyms } \\
& \text { bFGF }=\text { basic fibroblast growth factor } \\
& \mathrm{CM}=\text { conditioned medium } \\
& \text { dev-hAM }=\text { devitalized cryopreserved human } \\
& \text { amniotic membrane } \\
& \text { DMEM }=\text { Dulbecco's modified Eagle's medium } \\
& \mathrm{DPBS}=\text { Dulbecco's phosphate-buffered } \\
& \quad \text { saline } \\
& \mathrm{EBM}=\text { endothelial basal medium } \\
& \mathrm{EGM}=\text { endothelial growth medium } \\
& \mathrm{ELISA}=\text { enzyme-linked immunosorbent assay } \\
& \mathrm{FBS}=\text { fetal bovine serum } \\
& \mathrm{hAM}=\text { human amniotic membrane } \\
& \mathrm{HUVEC}=\text { human umbilical vein endothelial } \\
& \text { int-hAM }=\text { viable intact cryopreserved human } \\
& \mathrm{PDGF}=\text { platelet-derived growth factor } \\
& \mathrm{SD}=\text { standard deviation } \\
& \mathrm{TL}=\text { tissue lysates } \\
& \mathrm{VEGF}=\text { vascular endothelial growth factor }
\end{aligned}
$$

